of the Radio Industry Council, has submitted a minority report which is printed in full after the majority report, together with a point-by-point reply by the other members of the Committee to Mr. Stanley's views.

The main feature of Mr. Stanley's arguments is that he does not accept the assumption that veryhigh-frequency broadcasting need necessarily be introduced in the first place, and that, if it were, the experience of other countries that have introduced it, most of them using frequency-modulation, is decidedly unfavourable. If the plans for very high frequencies go ahead, then the general public will have to start considering buying a completely new receiver, the cost of which for an amplitude-modulator set would $\mathrm{be}$, on the average, $£ 20$ and for an equivalent frequency-modulation one £30. Mr. Stanley thinks that the future for the existing broadcasting systems is not necessarily hopeless and that the Committee's first task should be to investigate what may be done to improve them rather than replace them. The reply of the majority of the Committee is that veryhigh-frequency broadcesting is an unwelcome but inescapable complication, and that its introduction will probably be spread over at least ten years. During that time existing sets owned by the public will tend to wear out and need replacement, and the higher cost of $a_{b}$ frequency-modulation receiver is more than outweighed by the advantages of the system.

As in the case of the report on television, the Committee's terms of reference are for the most part concerned with technical problems; but the expansion of television and the introduction of very-highfrequency broadcasting both involve heavy capital outlay by the Government and the development by industrý of a wide range of new products, together with the necessity of the general public to find the money for purchasing these products. These are grave economic factors and will, no doubt, be an important, if not over-riding, consideration in any Government decision on these matters.

\section{HORIZONTAL DISPOSITION OF RHIZOMES}

$\mathrm{T}$ THE diageotropic disposition of the rhizome in bishop's weed (Aegopodium podograria) has been attributed by T. A. Bennet-Clark and N. G. Ball (J.Exp. Bot., 2, 169; 1951) to the action of two hormones working in opposition to one another; both are affected by gravity, and the horizontal position of the rhizome is due to an equilibrium between the effects of the two hormones.

N. G. Ball has now taken the investigation of the mechanism of this intriguing problem-the horizontal disposition of rhizomes - a stage further by examining the effects of several known growth-regulating substances on Aegopodium rhizomes (J. Exp. Bot., 4, $349 ; 1953)$. The experimental treatments, which involved the immersing of rhizomes for periods of forty-five minutes in solutions of the growthregulating substances, were carried out in the absence of visible light, the rhizomes being photographed every hour by infra-red radiation. After immersion in $10^{-4} M \beta$-indoleacetic acid the rate of rhizome growth is approximately doubled; but about five hours later it declines to one-third or less of its original value and then gradually recovers.
'This depression in the growth-rate can, however, be reversed by a second auxin treatment, the inference being that the exhaustion of some substance necessary for growth is not involved. It is suggested that the auxin which first accelerates elongation may later be converted into, or may cause the production of, an inhibitor. Thus, when successive immersions in auxin are repeated every two hours, the inhibition can be partially overcome as long as the treatment is continued. As the immersion of horizontal rhizomes in the growth-regulating substance also causes them to turn up, this is taken as giving support to the hypothesis indicated above; that is, an equilibrium is disturbed.

Thus it would appear that when a rhizome is immersed in a solution of auxin there is some mechanism which causes the auxin to accumulate on the lower side. As this may also be assumed to happen to any auxin produced in the rhizome itself, a probable explanation appears to be that the horizontal position assumed by the rhizome results from the balancing of the excess hormone on the lower side of an opposing one. It is pointed out that if the suggestion that $\beta$-indoleacetic acid causes an acceleration of growth followed by an inhibitory effect, or that it may itself be converted into an inhibitor, should prove to be generally valid, it would afford a simple and satisfactory explanation of autotropism. The effects of $2: 4$-dichlorophenoxyacetic acid, ascorbic acid, $2: 3: 4$-triiodobenzoic acid and $2: 4$-dichloranisole were also investigated.

\section{STRUCTURES OF NATIVE AND MERCERIZED CELLULOSES}

\section{By DR. M. K. SEN and DR. S. C. ROY}

Indian Jute Mills Association Research Institute, Calcutta

YN a previous publication, Sen and Woods ${ }^{1}$ reported 1 the presence of two new equatorial reflexions in the diffraction pattern of native celluloses of various origins. Continuation of this work with more detailed experimentation has now made it possible to make certrin assertions with regard to the origin of one of the reflexions, $Y$. From X-ray photographs taken under various conditions of prevailing humidity, intensity and purity of radiation (including monochromatic radiation) and with different cellulose samples, such as ramie, jute, cotton, viscose and paper, it has been noted that the reflexion occurs in greater or less intensity in almost all ordinarily available cellulosic fibres, native or mercerized. Under conditions of $60-70$ per cent relative humidity and $70-75^{\circ} \mathrm{F}$. temperature, the sharpness, intensity and dispersion of the diffraction maximum are at least equal to those of certain other equatorial reflexions (Fig. 1). The observations regarding its relative intensity in different samples when compared against Meyer and Mark 101 are summarized in Table 1.
Samples
1. Defatted white jute
2. Commercially degummed ramie
3. Surgical cotton
5. Ordinary rayon
Pre-swollen rayon (treating
with ethylene diamine, wash- with ethylene diam
ing and drying)
6. Mercerized ramie
7. Mercerized jute

Table 1 Intensity
Slightly less than that of 101 reflexion. Less than that of 101. Less than that of 101 . Slightly less than that of 101 .

Greater than that of 101. Greater than that of 101 (mer-
cerized). 